\title{
Objets frontières et brokering dans les négociations en recherche orientée par la conception
}

Boundary Objects and Brokering in Negotiations Within Design Based Research

Réjane Monod-Ansaldi, Caroline Vincent et Gilles Aldon

\section{OpenEdition}

Journals

Édition électronique

URL : https://journals.openedition.org/educationdidactique/4074

DOI : 10.4000/educationdidactique.4074

ISSN : 2111-4838

Éditeur

Presses universitaires de Rennes

\section{Édition imprimée}

Date de publication : 30 novembre 2019

Pagination : 61-84

ISBN : 978-2-7535-8012-1

ISSN : 1956-3485

Référence électronique

Réjane Monod-Ansaldi, Caroline Vincent et Gilles Aldon, « Objets frontières et brokering dans les négociations en recherche orientée par la conception », Éducation et didactique [En ligne], 13-2 | 2019, mis en ligne le 01 janvier 2021, consulté le 12 octobre 2022. URL : http://journals.openedition.org/ educationdidactique/4074 ; DOI : https://doi.org/10.4000/educationdidactique.4074 


\title{
OBJETS FRONTIÈRES ET BROKERING DANS LES NÉGOCIATIONS EN RECHERCHE ORIENTÉE PAR LA CONCEPTION
}

\author{
Réjane Monod-Ansaldi \\ Institut Français de l'Éducation - ENS Lyon \\ Caroline Vincent \\ Aix Marseille Univ, CNRS, LEST, Aix-en-Provence \\ Gilles Aldon \\ Institut Français de l'Éducation - ENS Lyon
}

\begin{abstract}
Cette étude s'intéresse au processus de négociation conversationnelle entre des enseignants et des chercheurs lors de réunions de conception collaborative de ressources pédagogiques numériques. À travers deux corpus d'interactions verbales donnant lieu à des négociations, nous observons de quelles façons des objets frontières sont définis et coconstruits par les participants, comment se développent les épisodes de brokering et explorons les conséquences de ces négociations pour les recherches, les acteurs et la production de savoirs.
\end{abstract}

Mots-clés : co-conception, collaboration, objet frontière, négociation, pédagogie.

\section{Boundary objects and brokering in negotiations within design based research}

This study investigates the process of conversational negotiation between teachers and researchers in collaborative design meetings of digital learning resources. Through two corpus presenting verbal interactions giving rise to negotiations, we observe how boundary objects are defined and co-constructed by the participants and how do brokering episodes evolve in the dialogues. We describe the consequences of these negotiations for research, for the actors and for the production of knowledge.

Keywords: co-conception, collaboration, boundary objects, negotiation, pedagogy.

Les auteurs remercient les actrices et acteurs des projets dont les réunions ont été analysées pour cet article d'avoir accepté d'être enregistré.e.s et d'avoir échangé avec eux sur les analyses produites. 


\section{OBJETS FRONTIÈRES ET BROKERING DANS LES NÉGOCIATIONS EN RECHERCHE ORIENTÉE PAR LA CONCEPTION}

Réjane Monod-Ansaldi, Caroline Vincent E Gilles Aldon

\section{INTRODUCTION}

Plusieurs courants méthodologiques de recherche en éducation s'appuient sur la collaboration entre des enseignants et des chercheurs. En résonance avec l'approche ethnologique proposée par Germaine Tillion dès les années 1930 (Tillion, 2009), ces méthodologies collaboratives visent à travailler « avec », et non plus «sur » les enseignants. Elles impliquent la co-construction d'une démarche de recueil et d'analyse de données pour l'investigation d'une question en lien avec les pratiques enseignantes (Desgagné, 1997), et un va-et-vient permanent entre recherche et intervention sur le terrain professionnel (Vinatier $\&$ Morissette, 2015). Ces travaux considèrent généralement l'enseignant comme un enseignant-chercheur (Elliott, 1986, 1990) ou un praticien réflexif (Shön, 1993) et entrent dans le cadre de recherche agissante (Giddens, 1987), ou de recherche de faisabilité à visées pragmatiques (Astolfi, 1993).

Ces différents courants correspondent aux recherches actions (Lewin, 1946), au Design experiment (Brown, 1992), à l'ingénierie didactique (Chevallard, 1982; Artigue 1992), ou au Design based research (Design based research Collective, 2003) ou recherche orientée par la conception. Cette dernière revendique une nécessité épistémologique pour les chercheurs d'agir avec les enseignants (Sensevy, Forest, Quilio \& Morales, 2013; Sanchez $\&$ Monod-Ansaldi, 2015; Sensevy, 2016). En effet, la complexité des situations d'enseignement apprentissage implique une approche systémique, mobilisant une pensée complexe, telle que définie par Morin (1990), en dialectique entre les points de vue de différents acteurs. Ces positions peuvent être mises en relation avec les limites des impacts des réformes basées sur l'evidence based education et la nécessité affirmée, pour transformer les systèmes éducatifs, de travailler avec les acteurs impliqués non seulement sur les innovations à mettre en place, mais sur les conditions de leur mise en œuvre (Bryk, 2015).

Les recherches orientées par la conception articulent des visées pragmatiques et heuristiques en développant une méthodologie collaborative, itérative, flexible, conduite en conditions écologiques (Barab \& Squire, 2004). La collaboration concerne toutes les étapes de la recherche, de la problématisation au recueil et à l'analyse des données. Le travail itératif permet de raffiner autant les dispositifs conçus que les modèles théoriques qui les sous-tendent, en fonction des résultats des analyses de données recueillies à chaque itération (Collins, Joseph \& Bielaczyc, 2004).

Les bénéfices attendus de ce type de recherche sont une connexion des problématiques traitées aux questions du terrain (Nieven, 2007), une meilleure prise en compte des conditions effectives d'enseignement et d'apprentissage (Barab \& Squire, 2004), la co-construction de savoirs articulant théorie et pratique, ou dépassant leur opposition (Sensevy, 2013), le développement professionnel des acteurs impliqués (Barab \& Squire, 2004) et une meilleure adaptation des ressources produites aux besoins (Wang \& Hannafin, 2005). Certaines de ces hypothèses de travail ont été soumises à l'analyse, notamment par Sensevy et al. (2013) qui présentent les ingénieries coopératives comme une variante particulière de Design Based research. Sanchez et MonodAnsaldi (2015) proposent d'appliquer à l'analyse de travaux de recherche collaborative orientée par la conception le cadre de la transposition métadidactique (Arzarello et al., 2014) qui articule les concepts d'objet frontière (Star \& Grisemer,1989) de brokering (Wenger, 1998) et de praxéologie (Chevallard, 1985), décrivant les activités humaines au travers de techniques pour résoudre certaines tâches et justifiées par un discours et une théorie. Plusieurs analyses (Monod-Ansaldi \& Gruson, 2016; Aldon, Cusi, Morselli, Panero \& Sabena, 2017; Sanchez, Monod-Ansaldi, Vincent \& Safadi, 2017) se sont ainsi été focalisées sur les interactions autour d'objets frontières entre acteurs issus de différentes institutions, au sens de Chevallard (2003), qui appelle institution tout dispositif social permettant et imposant des manières de faire et de penser qui lui sont propres. Notre démarche se situe dans la continuité de ces travaux.

EducTice est une équipe pluridisciplinaire de l'Institut français de l'éducation de l'ENS de Lyon, dont plusieurs membres appartiennent à l'EA S2HEP de l'Université Lyon 1 ; elle étudie les opportunités du numérique pour la rénovation de l'enseignement des mathématiques et des autres disciplines scientifiques, pour l'évolution des pratiques pédagogiques de l'enseignement supérieur, et pour la formation des enseignants. Elle explore également les apports des travaux collectifs enseignants, en particulier à travers l'analyse des évolutions de leurs systèmes de ressources. Certains travaux d'EducTice passent par la conception de dispositifs d'enseignement et/ou 
d'apprentissage au sein de recherches collaboratives et pluri-partenariales. Ils impliquent des chercheurs, des enseignants, des formateurs, des ingénieurs pédagogiques, des informaticiens, des membres d'entreprises, des conseillers pédagogiques du supérieur et des responsables institutionnels. D'un point de vue méthodologique, ces travaux relèvent de la recherche orientée par la conception. Ils s'appuient en particulier sur un laboratoire d'innovation pédagogique et numérique (LIPn, Sanchez, 2015a), où sont développés des outils de créativité, de collaboration et de conduite de projet.

La question des modalités plus fines de mise en œuvre de travaux collaboratifs impliquant différentes communautés de pratiques (Wenger, 1998) au sein de différentes institutions, est ainsi partagée par une part importante de l'équipe EducTice, qui a saisi sa richesse pluri catégorielle et pluridisciplinaire pour mettre en œuvre une analyse collaborative de ses propres travaux. En effet, les principes des recherches collaboratives insistent sur l'importance de constituer des groupes où des points de vue différents se côtoient; mais comment ces différents points de vue se rencontrent-ils durant ces recherches? Comment s'expriment-ils, s'opposent-ils? Comment sont-ils négociés entre les acteurs durant les travaux? Et quelles sont les conséquences de ces négociations pour les recherches, les acteurs, les objets et les savoirs produits? Ces questions sont explorées depuis 2016, au sein d'un séminaire interne à l'équipe EducTice visant à mieux comprendre les processus en œuvre dans la collaboration, en particulier par l'étude des interactions qui se développent lors de réunions pluri catégorielles au sein de projets collaboratifs.

Nous présentons ici des éléments issus de ces analyses pour deux projets de recherche différents. Elles permettent de caractériser dans les échanges entre participants les épisodes de négociation de sens autour de désaccords explicites ou non, et leurs conséquences pour les acteurs, les travaux, et les savoirs en construction. Après un exposé du cadre des négociations interactionnelles, et des concepts d'objets frontière et de brokering au sens où ils sont travaillés dans le cadre de la transposition métadidactique (Arzarello et al., 2014 ), nous apportons une description rapide du projet de séminaire, et présentons les contextes des deux projets étudiés, les corpus qui en sont issus et la méthodologie mise en œuvre pour leur analyse. Les résultats obtenus sont ensuite rapportés et discutés.

\section{NÉGOCIATIONS, OBJETS FRONTIÈRES ET BROKERING}

Pour mieux comprendre les processus en œuvre dans les collaborations de recherche orientée par la conception entre acteurs des communautés d'enseignants et de chercheurs, nous focalisons dans cet article notre regard sur les interactions qui se développent lors des réunions mobilisant ces différents acteurs. Nous présentons ici le cadre de l'analyse conversationnelle utilisé pour repérer et décrire les négociations qui s'y produisent et les concepts d'objet frontière et de brokering qui permettent de saisir des processus de partage et d'internalisation entre groupes issus d'institutions différentes et d'en étudier les conséquences sur l'avancée des travaux et la construction collective de savoirs.

\section{Négociations interactionnelles}

La description des négociations interactionnelles s'inscrit dans le domaine de l'analyse conversationnelle (Sacks, 1963; Schegloff, 1974). Il s'agit de l'étude des mécanismes et de l'organisation du discours en interaction, en prenant en compte la complexité du contexte et à partir de données authentiques.

Kerbrat-Orecchioni (2001) définit la négociation interactionnelle comme

« un processus interactionnel plus ou moins local, susceptible d'apparaître dès lors qu'un différend surgit entre les participants concernant tel ou tel aspect de l'interaction et ayant pour finalité de résorber ce différend » (p. 399).

Pour qu'il y ait négociation, il faut donc qu'il y ait un désaccord, puis que les participants œuvrent ensemble pour aboutir à un accord. La négociation se différencie donc de la dispute (dans laquelle chacun affirme sa position sans chercher un accord) et de la simple discussion (dans laquelle deux points de vue divergents coexistent de façon pacifique). Ainsi, dans cet exemple illustratif proposé par Traverso 
(2004), la négociation commence au troisième tour de parole, lorsque le désaccord se cristallise :

tour de parole 1 : A fait une proposition à B. Par exemple, « Elsa arrive à la gare à $17 \mathrm{~h}$ »;

tour de parole 2 : B la conteste et fait une contreproposition. Par exemple, « ah non elle arrive à $15 \mathrm{~h} »$.

Il y a alors deux possibilités au troisième tour de parole : soit un ajustement, soit la cristallisation du désaccord qui entraînera alors une négociation. Concernant l'ajustement, les participants peuvent arriver à un accord immédiat :

tour de parole 3 : (« ah bon d'accord, elle arrive à $17 \mathrm{~h} »)$.

Ils peuvent également ne pas thématiser le désaccord :

tour de parole 3 : «peu importe, dans tous les cas il faudra aller la chercher $»$.

En cas de cristallisation du désaccord, le premier locuteur rejette la contre-proposition et c'est alors que la négociation débute :

tour de parole 3 : « ah non je suis bien certain qu'elle arrive à $17 \mathrm{~h} »$.

La négociation conversationnelle peut porter sur tout ce qui se rapporte à la situation : le script de l'interaction, l'alternance des tours de parole, la relation entre les interlocuteurs; les thèmes traités, l'identité, le sens, le contrat de communication ${ }^{1}$.

Nous nous appuyons sur la description de Kerbrat-Orecchioni (2001) pour identifier les négociations. D'après l'auteure, une négociation conversationnelle nécessite :

- (1) au moins deux négociateurs directement impliqués dans le désaccord qui peuvent être assistés / remplacées par des tiers ainsi qu'un médiateur éventuel (prévu comme tel ou qui émerge dans l'interaction);

- (2) un objet à négocier;

- (3) un état initial de divergence;

- (4) la mise en ouvre de procédures pour tenter de résorber le désaccord;

- (5) un état final (réussite si ralliement ou compromis; échec si piétinement ou dispute) (Kerbrat-Orecchioni, 2004).
Les négociations sont la plupart du temps implicites, c'est-à-dire que le désaccord n'est pas thématisé. Il arrive également que la négociation soit menée sur un mode plus explicite, lorsque les interactants utilisent des énoncés métacommunicatifs du type « je ne suis pas d'accord avec ce qui est proposé », « je ne souhaite pas que vous me tutoyez », ou encore « j'aimerais que l'on revienne sur un point qui ne me convient pas $»$.

Dans une situation de réunion de conception collaborative, on peut s'attendre à voir émerger de nombreuses négociations sémantiques et thématiques qui permettront aux différents acteurs de co-construire un objet commun.

\section{Objets frontières}

Le concept d'objet frontière est né dans un contexte d'étude ethnographique des mécanismes de coordination du travail scientifique (Star $\&$ Grisemer, 1989). Il repose sur trois caractéristiques essentielles : la flexibilité interprétative, la structure ou l'organisation matérielle et la granularité. L'objet frontière s'entend comme un dispositif permettant d'amorcer un travail commun entre plusieurs mondes et assurant une flexibilité suffisante pour que chaque acteur puisse trouver un intérêt à son étude ou à son usage. Il est à noter que cette interprétation, cet arrangement, ne peut concerner qu'un objet qui a une importance professionnelle dans chacune des communautés. La différence de perception provient des interprétations différentes de chaque communauté. Mais cet aspect, si essentiel soit-il pour la compréhension du concept, n'est pas suffisant à décrire l'objet frontière, comme le fait remarquer Star (2010, p. 603) : «Because it was in the right place at the right time, boundary objects became almost synonymous with interpretive flexibility ».

Les objets frontières focalisent les interactions et permettent de définir le concept d'internalisation : une propriété, un concept, une connaissance apportée par l'une ou l'autre des communautés seront internalisés lorsque ces éléments feront partie intégrante de l'objet frontière sur lequel se focalise le travail collaboratif. Les praxéologies modifiées par ce travail sur l'objet frontière sont ainsi partagées et amènent une compréhension élargie des phénomènes étudiés. «A Meta-Didactical Transposition 
produces a dynamic change in the praxeologies of the community of teachers. [...] Presumably, also, the resarcher praxeologies change as well, as a result of their encounters with the community of teachers " (Arzarello et al., 2014, p. 356).

Considérant la structure même de l'objet étudié, nous proposons de désigner par ce terme " objet " également le conteneur symbolique incluant diverses composantes, qui pourraient hériter des propriétés de l'objet conteneur. Cette proposition reprend l'approche de la programmation-objet, pour laquelle: "An object is something people (or in computer science, other objects or programs) act towards and with.» (Star, 2010, p. 604). Dans cette métaphore, les concepts d'encapsulation et d'héritage proposent une structure des éléments, propriétés et caractéristiques contenus dans l'objet frontière comme autant de composantes héritant de propriétés générales du « conteneur ». Ainsi, l'étude et le travail sur et avec les composantes permettraient d'inférer des propriétés de l'objet lui-même. Cette métaphore pointe aussi le fait que les objets n'existent que par les actions que les acteurs accomplissent sur ces objets, en interactions ou non avec les autres composantes. Cette structure des objets frontières est essentielle pour comprendre les discussions et débats qui ne portent généralement que sur quelques-unes de ses composantes (Star, 2010, p. 602). Elle permet également de considérer l'apport de la collaboration entre acteurs de points de vue différents pour la connaissance de l'objet frontière. Comme chacun agit sur et « fait exister » dans les échanges certaines composantes de l'objet, l'activité collective ouvre la possibilité de construire des savoirs sur l'ensemble des composantes reconnues par les acteurs présents, et d'en identifier de nouvelles, dans leurs relations et leur articulation.

Dans ces conditions, les types d'activité conduits sur les objets-frontières, ou leurs composantes, marquent la relation des acteurs à cet objet. Trompette et Vinck (2009) à la suite de Carlile (2004) proposent trois types d'activité qui sont susceptibles de modifier les frontières en élargissant l'espace de compréhension partagée de l'objet par le groupe / pour les différentes communautés :

- le transfert;

- la traduction;

- la transformation.

Le transfert de connaissances porte sur des communications dont les locuteurs ne contestent pas la validité et pour lesquelles la frontière demeure stable. Il s'agit d'interactions qui peuvent produire des désaccords, mais pour lesquelles les échanges concernent des aspects concrets, syntaxiques de l'objet frontière, sans aborder les aspérités qui pourraient se jouer pour articuler des composantes différentes identifiées par les différentes communautés.

La traduction s'appuie sur une approche sémantique où la frontière se déplace dans une négociation du sens de certaines composantes sur lesquelles les différents acteurs agissent. La recherche d'un sens commun, partagé conduit à une négociation où la frontière se déplace au sein d'interactions qui concernent le sens de certaines composantes, et qui aboutissent à des changements de points de vue, l'objet agissant comme médiateur cognitif entre les communautés mais aussi entre les institutions représentées.

La transformation est associée à une médiation sociale dans laquelle les intérêts des institutions construisent pragmatiquement l'espace frontière qui peut être reconnu socialement entre les institutions. Les connaissances en jeu sont alors transformées par la négociation entre les acteurs dans une création d'un nouveau savoir partagé utilisable dans les pratiques de chaque communauté : "When interests are in conflict, the knowledge developed in one domain generates negative consequences in another. Here the costs for any actor are not just the costs of learning about what is new, but also the costs of transforming "current" knowledge being used (i.e., common and domain-specific knowledge) »(ibid, p. 559).

Nous utilisons ce concept d'objet frontière à l'origine d'une relation professionnelle entre plusieurs communautés liées à différentes institutions, pour expliquer les moteurs de l'évolution des relations entre les différentes communautés en lien avec l'internalisation de praxéologies et repérer les composantes de l'objet frontière qu'elles mobilisent lors des phases de négociations. Nous cherchons ainsi à identifier si des activités de transfert, de traduction ou de transformation prennent place dans les processus de négociation, et interrogeons leurs conséquences pour les différents acteurs et pour l'évolution du partage de l'objet-frontière tant d'un point de vue syntaxique que sémantique et pragmatique. 


\section{OBJETS FRONTIÈRES ET BROKERING DANS LES NÉGOCIATIONS EN RECHERCHE ORIENTÉE PAR LA CONCEPTION}

Réjane Monod-Ansaldi, Caroline Vincent E Gilles Aldon

\section{Broker et brokering}

Le broker, ou courtier de connaissances est défini comme une personne connaissant suffisamment deux communautés pour pouvoir transférer des éléments d'une pratique de l'une vers l'autre, et ouvrir des possibilités de compréhension entre ces communautés (Wenger, 1998). Dans le cadre de la transposition métadidactique (Arzarello et al., 2014), il est vu comme favorisant la compréhension approfondie d'objets frontières et le partage de praxéologies dans le processus d'internalisation.

La littérature sur les brokers et le courtage de connaissances concerne surtout le domaine de la santé publique où des brokers professionnels apparaissent, et où des moyens sont déployés pour favoriser la mobilisation des savoirs issus de la recherche autant par les praticiens que par les usagers ou les décideurs politiques. Les revues de Munerol, Cambon et Alla (2013) et Ridde et Dagenais (2013) identifient des conditions nécessaires au courtage de connaissances, telles que la crédibilité du broker, sa double légitimité dans les deux communautés, des actions sur un temps long, et un soutien institutionnel. Elles insistent également sur son rôle dans la genèse d'un langage commun entre les deux communautés, en lien avec les actions de transfert, et avec la construction de praxéologies partagées présentée dans le paragraphe précédent. Des compétences analytiques, pour lire comprendre et évaluer des résultats de recherche, des compétences de communication, une flexibilité, une adaptabilité, une bonne compréhension des cultures des deux communautés et la production d'un langage adapté à chacune d'elles sont également notées dans la revue de Mallidou et al. (2017). Mais ces publications tendent à personnifier la fonction de broker, en lien avec la problématique de professionnalisation, alors que nos analyses de projets de recherche collaborative la montrent fréquemment comme plus dispersée, endossée par une personne ou l'autre au cours des travaux, lors de ce qu'on peut nommer plutôt des épisodes de brokering (Nizet \& Monod-Ansaldi, 2017; Aldon \& Panero, 2017).

Dans le cadre de cet article, nous explorerons les liens entre négociation et brokering, en tentant de préciser si des composantes particulières d'objets frontières sont la cible de processus de brokering au cours des phases de négociation identifiées, et en cherchant quel type d'activité le broker met alors en œuvre.

\section{MÉTHOdOLOGIE}

\section{Séminaire d'analyse des recherches} collaboratives d'EducTice

Créé en 2016, le séminaire bimestriel d'analyse des recherches collaboratives d'EducTice réunit des membres de l'équipe : enseignants du primaire et du secondaire, ingénieurs pédagogiques, enseignants chercheurs de différentes disciplines (didactique, mathématiques, psychologie, sciences du langage, sciences de l'information et de la communication, ingénierie pédagogique), et des doctorants travaillant tous dans le domaine de l'éducation et du numérique. Il se présente comme un lieu d'échanges épistémologiques et méthodologiques. Les modalités de travail sont les suivantes :

- en amont du séminaire, un membre de l'équipe met à disposition un corpus issu d'un projet de recherche collaboratif (vidéo de réunion, verbatim...), contextualisé par une courte présentation, ce qui permet aux autres participants d'en prendre connaissance et éventuellement de l'analyser;

- lors du séminaire, au moins deux analyses de ce corpus sont présentées par une personne ayant participé au projet (analyse interne) et une personne extérieure (analyse externe) - il s'en suit un travail collectif pour discuter les cadres et méthodologies mobilisés, les résultats obtenus, et leurs apports pour la pratique de travaux de recherche collaboratifs au sein de l'équipe;

- en aval du séminaire, les participants remplissent un questionnaire réflexif sur la séance qui vient d'avoir lieu.

L'objectif du séminaire est de co-construire au sein de l'équipe des outils théoriques et méthodologiques qui s'appuient à la fois sur l'expérience de terrain et sur des analyses scientifiques de traces de cette expérience.

Dans le cadre de cet article, nous nous appuyons sur deux séances du séminaire qui ont eu lieu au cours de l'année 2016-2017, dont les corpus permettent de distinguer des séquences de négociations de façon très saillante. Elles concernent des réunions collaboratives au sein de deux projets différents. Nous proposons dans le paragraphe suivant une description succincte des deux projets concernés, des équipes impliquées et des corpus analysés. 


\section{Contextes des projets collaboratifs étudiés et corpus analysés}

\section{Le projet Pédagogies Immersives}

Le collège Fontreyne de Gap est impliqué depuis plusieurs années dans des projets liés aux usages du numérique dans l'enseignement. Ces projets associent expérimentation, innovation pédagogique et collaboration avec des laboratoires de recherche (pour exemple le projet européen FaSMEd ${ }^{2}$ ). Le projet actuel est né de ce contexte et de la rencontre avec la société Immersive-Colab qui développe une plateforme de réalité virtuelle. Ainsi, le collège virtuel a vu le jour comme espace immersif numérique dans lequel chaque acteur est représenté par un avatar qui peut se déplacer dans un environnement 3D. Les perspectives pédagogiques sont apparues riches et diversifiées et après une première expérimentation avec un enseignant du collège, le projet a intégré le réseau des Lieux d'éducation associés à l'IFE (LéA ${ }^{3}$ ) en septembre 2016. Le projet cherche à tirer le meilleur profit de cet environnement numérique pour les apprentissages des élèves et vise à :

- évaluer les apports pédagogiques d'un tel espace immersif;

- étudier les incidences d'espaces immersifs sur l'enseignement et l'apprentissage des disciplines, notamment en mathématiques;

- étudier le redimensionnement du territoire éducatif en relation avec les usages d'un espace immersif, notamment développer de nouvelles passerelles pédagogiques entre le premier et le second degré;

- évaluer l'impact d'un espace immersif et de la construction d'une communauté de pratique sur le rapport au savoir.

Ce travail se conduit en collaboration avec l'équipe éducative du collège mais aussi avec des enseignants des écoles primaires associées. Le Laboratoire d'Économie et de Sociologie du Travail, (CNRS, Université Aix-Marseille) participe également au projet en traitant en particulier des questions d'ergonomie, qui ne seront pas abordées dans cet article.

Le corpus étudié correspond à une des premières réunions plénières du projet regroupant les enseignants susceptibles d'y participer, le chef d'établissement, les chercheurs impliqués dans le projet et l'ingénieur qui a développé le collège virtuel. Si l'on réfère aux rôles définis par des chartes au sein du réseau des LéA ${ }^{4}$, sont en particulier présents à cette réunion, le correspondant IFE chercheur responsable des recherches menées et des liens avec l'IFE, le correspondant LéA qui contribuent à l'animation des travaux et à leur insertion dans l'établissement, en particulier par des relations avec le chef d'établissement et par des actions de communication et le référent du comité de pilotage qui accompagne le développement de ce LéA. Toutes les institutions parties prenantes de ce projet sont ainsi présentes et les enjeux de cette réunion concernent l'acceptabilité du projet et des collaborations futures.

Lors du séminaire de l'équipe EducTice, les données mises à l'étude étaient constituées du verbatim de la réunion. L'analyse interne, réalisée par une personne participant au projet mobilisait le cadre de la transposition didactique. L'analyse des négociations à l'œuvre dans la réunion a été menée par une personne extérieure au projet. Dans la suite, des observations en classe et à travers la plateforme ont été réalisées, analysées et discutées avec les enseignants. Nous nous référerons à ces données complémentaires dans la discussion.

\section{Le projet École Numérique et Industrie (ÉNI)}

ÉNI est un projet lauréat du programme "Culture scientifique, technique et industrielle et égalité des chances» (Agence nationale de rénovation urbaine), piloté par le Réseau Canopé en collaboration avec l'IFÉ. Il vise la conception d'une plateforme de ressources pédagogiques pour le développement de la culture scientifique et technologique des élèves.

$\mathrm{Au}$ sein du projet, l'équipe EducTice a conçu un jeu épistémique numérique (Sanchez, 2015b) nommé Paye ta preuve, destiné à la diffusion de la culture scientifique, technique et industrielle (CSTI). Il s'agit d'ouvrir les perspectives d'orientation professionnelle des collégiens en les sensibilisant à la CSTI, en particulier dans ses aspects trop peu souvent évoqués concernant les sciences et les techniques industrielles. Une attention particulière est portée à l'intégration de certains publics souvent laissés en marge de la CSTI tels que les filles, les élèves en situation de handicap et les élèves socialement défavorisés.

La méthodologie mise en œuvre pour ce projet dans le cadre du laboratoire d'innovation pédagogique et numérique, correspond à une recherche 
collaborative orientée par la conception (Wang \& Hannafin, 2005). L'équipe de conception est composée de deux chercheurs, d'un ingénieur pédagogique, de cinq enseignants de collège de différentes disciplines, d'un développeur informatique et d'intervenants ponctuels (collégiens, graphiste, game designer, chercheurs, professionnels de la CSTI, architecte de l'information et catera).

Le corpus étudié en septembre 2016, correspond au verbatim d'une réunion de l'équipe de conception du jeu, qui a eu lieu trois mois après le début $\mathrm{du}$ projet et qui rassemblait quatre enseignants de collège, un enseignant-chercheur spécialiste du domaine des sciences et techniques industrielles, une chercheuse et un ingénieur pédagogique. Cette réunion visait en particulier à concevoir le scénario et la métaphore du jeu (Sanchez, 2015b).

Dans ce cas, lors du séminaire, l'analyse interne s'est focalisée sur l'analyse conversationnelle des négociations et l'analyse externe mobilisait le cadre de la transposition métadidactique Des données complémentaires correspondant à des entretiens menés auprès des enseignants participants, et à la version finale du jeu présentée dans le rapport de fin de projet (Benech \& Vincent 2017) ont été utilisées pour la discussion.

Les analyses réalisées dans les deux séminaires ont mis en valeur la complémentarité des approches et la compatibilité de ces cadres théoriques (négociation, objet frontière et brokering) et nous ont incités à approfondir ce double regard par la méthode que nous présentons dans le paragraphe suivant.

\section{Méthode d'analyse des verbatims}

Les deux verbatims de réunion analysés dans le cadre des deux séances de séminaires ont été repris pour identifier les phases de négociation de sens entre les participants, en tenant compte des éléments qui avaient été repérés par les analyses internes et externes présentées lors du séminaire.

Dans l'analyse conversationnelle francophone (Kerbrat-Orecchioni, 1990; Maingueneau, 1996), on considère les unités de description dialogales (construites par au moins deux locuteurs) et monologales (construites par un seul locuteur), et l'interaction se décompose en séquences, les séquences en échanges, les échanges en interventions et ces dernières en actes de langage.
Dans les unités dialogales, l'interaction est l'unité de rang supérieur. Elle est définit comme «ce qui se passe entre la mise en contact des participants et leur séparation »(Traverso, 1999, p. 38). La séquence est considérée comme un «bloc d'échanges reliés par un fort degré de cohérence sémantique et/ou pragmatique » (KerbratOrecchioni, 1990 p. 218). Les séquences peuvent être imbriquées les unes dans les autres, les plus courantes étant les séquences d'ouverture et de clôture encadrant le corps de l'interaction. L'échange est la plus petite unité dialogale. Elle est composée de deux interventions au minimum : une initiative ( «bonjour ça va? ») et une réactive ( " très bien et toi?»), suivis la plupart du temps par une évaluation ( «impeccable ») puisque les échanges à structure ternaire sont les plus fréquents.

Concernant les unités monologales, un échange est composé d'une ou plusieurs interventions produites par un seul locuteur. Une intervention est composée d'au moins un acte de langage, qui est « la plus petite unité réalisant par le langage une action (ordre, requête, assertion, promesse, information...) destinée à modifier la situation des locuteurs » (Maingueneau, 1996). Les actes de langage sont le noyau de l'interaction et ils peuvent être multiples au sein d'une même intervention, par exemple « auraistu un mouchoir? » contient un acte de langage direct (la question littérale) et un indirect (la requête d'un mouchoir).

Nous nous appuyons sur ces unités dans les analyses qui suivent pour décrire les deux interactions au centre de notre étude.

Pour comprendre comment les négociations mobilisent les objets frontières et le brokering, les composantes d'objets frontières en discussion dans les groupes ont été repérées pour les différentes communautés dans les verbatims découpés en séquences. Les activités des participants sur ces composantes ont été catégorisées en transfert, traduction ou transformation, en fonction de la nature des échanges observés.

Les interventions de médiations entre points de vues de différentes communautés ont aussi été isolées et analysées comme des épisodes de brokering : qui intervient? Comment? En rapport avec quelle composante de l'objet frontière? 


\section{OBJETS FRONTIÈRES ET BROKERING AUX DIFFÉRENTES SÉQUENCES DE NÉGOCIATION}

\author{
Réunion de début de projet \\ Pédagogies Immersives
}

Rappelons que l'interaction dont il est question est une première réunion entre des partenaires potentiels (enseignants, dont le correspondant LéA, chef d'établissement, chercheurs dont le correspondant IFE, référent numérique, et le référent du comité de pilotage des LéA) impliqués dans un projet pédagogique qui consiste à mettre en place un collège virtuel. Le matin précédant cette réunion, les enseignants ont testé le collège virtuel en conditions réelles dans leurs classes. Le verbatim de la réunion a été découpé en quatre séquences.

\section{La séquence d'ouverture}

Analyse de l'interaction

L'interaction se découpe en quatre séquences :

$\mathrm{Au}$ tout début de l'interaction, le chercheur, correspondant IFE, intervient pour proposer le déroulé suivant :

« ça serait bien si vous pouviez nous expliquer un peu ce qui a été fait. Comment vous en êtes arrivé là dans un premier temps, et ensuite comment vous avez vécu cette matinée. Et puis éventuellement en continuant en se disant comment vous pourriez continuer [...]. Je vous laisse la parole».

Suite à cette intervention initiative, on s'attend donc à des interventions réactives répondant à ces trois thèmes :

- les usages liés au numérique passés;

- les impressions concernant les usages de la matinée;

- les usages possibles dans le futur.

C'est en effet le cas de la première intervention, puisque un premier enseignant répond en respectant les trois thèmes proposés, ratifiant ainsi la proposition du chercheur. Pour chacune de ses réponses, il précise deux sous-thèmes qui pour lui sont indissociables: les usages pédagogiques et les difficultés techniques. En effet, dans ce contexte, les usages pédagogiques sont fortement contraints par la technique :

« on se posait déjà la question de est-ce que le but c'est d'utiliser l'outil ainsi ? et en même temps y'avait quand même des problèmes techniques dans cette utilisation »; " j'aurais aimé faire travailler les élèves sur l'algorithmique [...]. On avait essayé avec T. mais Scratch ça s'intègre pas bien dans le collège virtuel ».

L'introduction du thème «difficultés techniques » va induire la deuxième séquence de l'interaction correspondant à la négociation du déroulé des thèmes proposés par le chercheur.

\section{Objet frontière et brokering}

Dans cette réunion, l'objet sur lequel tous les participants focalisent leurs actions est le collège virtuel, qui tient la place d'objet frontière. Deux composantes de l'objet frontière sont évoquées durant la première séquence : d'une part l'objet technologique, plateforme numérique utilisant des logiciels spécifiques qui permettent à travers un réseau de la parcourir avec un pointeur avatarial; et d'autre part, la composante pédagogique de cette plateforme avec les outils spécifiques permettant une scénarisation d'une séance avec des élèves. Les deux composantes sont imbriquées mais les acteurs focalisent leurs interventions sur le fonctionnement technique du collège virtuel qui apparaît comme une composante essentielle. Cette séquence d'ouverture se caractérise ainsi par une activité de transfert. Il s'agit d'abord d'accepter la discussion sur les composantes proposées de l'objet frontière (objet technologique et potentialités pédagogiques) puis de déterminer la facette de l'objet technologique sur laquelle il est possible d'agir et pour laquelle les points de vue des acteurs peuvent s'accorder. En effet, l'objet technologique collège virtuel aurait pu être abordé d'un point de vue conceptuel en considérant les particularités techniques de la plateforme immersive en opposition, par exemple, avec les espaces numériques de travail dont les enseignants sont familiers. Mais la discussion porte sur les difficultés concrètes du déploiement de la plateforme dans les contextes des écoles et du collège. L'action sur l'objet relève ainsi d'un phénomène de transfert, l'accord pourra alors se concrétiser à travers la reconnaissance institutionnelle des problèmes qui se posent. 


\section{OBJETS FRONTIÈRES ET BROKERING DANS LES NÉGOCIATIONS EN RECHERCHE ORIENTÉE PAR LA CONCEPTION}

Réjane Monod-Ansaldi, Caroline Vincent E Gilles Aldon

\section{Séquence de négociation : le désaccord implicite des enseignants}

Analyse de l'interaction

Après l'intervention du premier enseignant, les interventions suivent toutes un même schéma : une réponse aux deux premiers thèmes initiés par le chercheur (usages passés et du matin), sans aller jusqu'au troisième thème. Les interventions des enseignants sont unanimes : on ne peut pas prévoir d'usages futurs sans résoudre d'abord les difficultés techniques.

Les instances en présence reconnaissent l'importance de cet aspect et cherchent à clore le sujet qui se situe hors de la proposition initiale :

Le chef d'établissement propose de remettre à plus tard l'évocation de ce thème invoquant une réunion qui doit se tenir dans quelques jours : " on pourra évoquer ce problème avec les décisionnaires ». Le chercheur également atteste de l'importance du thème : « c'est une partie matérielle importante [...] c'est un obstacle qu'il va falloir franchir pour avancer ».

Le tour de table reprend alors et de nouveau, l'obstacle technique est évoqué par les enseignants pour chaque usage pédagogique dont il est question, comme le résume cet enseignant : « l'ambition pédagogique est réduite par la réalité technique ».

Deux obstacles en particulier sont levés par les enseignants : les problèmes de connexion et de réseau et le matériel vétuste.

À ce stade de l'interaction, il est clair que tant que le sujet « technique » ne sera pas clôt, il ne sera pas possible de compléter le déroulé des thèmes proposés initialement, c'est-à-dire d'envisager des usages pédagogiques possibles dans le futur. C'est là que se joue la négociation. Nous considérons que celle-ci est implicite puisqu'aucune intervention ne verbalise le désaccord ni le nœud du problème, bien que celui-ci soit clairement identifiable pour l'analyste.

\section{Objet frontière et brokering}

L'activité de transfert se poursuit dans cette séquence sans aboutir réellement à un accord des participants, mais sans non plus de désaccord explicite. On observe plutôt une volonté de la part de tous les acteurs de voir plus clairement l'objet. La composante technique de l'objet collège virtuel paraît de mieux en mieux cernée, tous les acteurs comprennent l'enjeu et les pistes de solutions. En ce sens, l'action conjointe du correspondant LéA et du chef d'établissement permet de construire autour de cette composante un consensus sur les problèmes de fonctionnement et les remèdes à y apporter. En permettant de faire apparaître des solutions, ces deux acteurs jouent un rôle de broker qui facilite la compréhension syntaxique de cette composante de l'objet frontière. Durant cette activité de transfert, le brokering apparaît comme un acte d'explicitation de la description de la composante technique du collège virtuel. À aucun moment la discussion ne porte sur le concept même de plateforme immersive 3D, qui dépasse les seules techniques d'utilisation de la plateforme considérées par les problèmes qu'elles posent et qui doivent être résolus. La frontière est établie et les discussions se font dans l'espace reconnu par tous les locuteurs comme commun. Cette phase se termine sur un point technique «Bon effectivement après d'un point de vue technique je sais pas comment on rappelait la procédure qui avait été faite pour pouvoir mettre un clip » qui donne l'occasion au correspondant LéA, dans un rôle de broker, d'activer une discussion d'abord pragmatique « le moment où les partenaires viennent euh... ben comme aujourd'hui, ça crée une accélération sur la quinzaine de jours qui précèdent [...] donc ce qu'on a fait, c'est qu'on a déposé un des clips sur le collège virtuel », puis sémantique pour enrichir la frontière par une approche de traduction en évoquant une nouvelle façon d'agir sur la plateforme du collège virtuel en considérant ses potentialités pédagogiques.

\section{Séquence de négociation: les arguments des uns et des autres}

\section{Analyse de l'interaction}

Dans la séquence suivante, plusieurs intervenants proposent un certain nombre de solutions et d'arguments afin de dénouer la situation et de répondre aux arguments des enseignants :

- le chercheur tout d'abord indique que les problèmes tels qu'ils sont décrits par les enseignants peuvent ne pas être dus uniquement à la technique mais également à un manque de scénarisation pédagogique; 
- le correspondant LéA a un rôle particulier puisqu'il enseigne dans le collège et est en charge de l'organisation et de la mise en œuvre de l'expérimentation auprès des différents partenaires institutionnels. À l'un des enseignants qui fait valoir son manque de formation et la position délicate dans laquelle il est placé face aux élèves ( « tous ces problèmes techniques, nous on peut pas savoir, c'est difficile », « j'ai un élève qui s'est retrouvé coincé dans un arbre, euh... je sais pas! Comment je fais? »), il propose de mettre en place une formation pour l'ensemble des enseignants impliqués dans le projet à partir des problèmes observés.

Progressivement, les enseignants reprennent le tour de table et des usages pédagogiques sont proposés. Des arguments techniques sont de nouveau levés ( « après techniquement pour l'instant, ils ne sont pas capables de le faire ») mais ceux-ci sont vite écartés par des contre-arguments du correspondant LéA s'appuyant à la fois sur la technique et sur les usages observés : « on n'a jamais eu de problème de débit », « techniquement, j'ai fait le test », « actuellement, ce qui marche c'est à deux avec onze élèves », « je pense qu'avec une classe c'est possible ».

À partir de la description d'un usage en classe qui a été gêné par la technique, le référent du comité de pilotage des LéA, qui a aussi assisté aux expérimentations, fait valoir un nouvel argument :

«C'était très très intéressant à observer dans le primaire. Alors euh je vais pas nier, surtout pas qu'il est impératif dans un projet comme ça de lever tous les obstacles techniques et au plus haut niveau notamment le matériel et les questions de débit, c'est fondamental. Mais c'était assez intéressant de voir en terme de motivation, de l'activité et d'autonomie ce qui s'est déroulé dans la classe des CM1 CM2 où l'obstacle technique audio n'a pas été complètement contourné mais en tous cas n'a pas démobilisé les élèves. D'euxmêmes, ils sont partis sur le tchat, ce qui a posé un problème de tchat collectif qui ne leur permettait pas de voir où ils se plaçaient en tous cas par rapport à leur. interlocuteur binôme j'ai envie de dire donc la conversation était mélangée. Dès qu'ils ont eu le tchat individuel, pour ceux qui ont pu y accéder, ils s'en sont emparés, ils ont communiqué. Et on a vu des équipes se déplacer dans les salles de l'école pour aller prendre de l'information sur comment comment t'as fait, où est ce que tu as cliqué pour avoir, tu viens je t'aide.
Et aussi des groupes, c'était assez manifeste, qui sont allés, parce que y'avait effectivement cette difficulté de communication écrite alors qu'au cycle 3 enfin en CM1 CM2, on est plutôt sur de l'oral, chercher leur classeur outil pour avoir le vocabulaire et être capable de communiquer. Donc là, on était vraiment sur une dynamique très intéressante, je veux dire l'obstacle de premier niveau a été non pas levé mais contourné d'une certaine façon et avec sur la fin de l'heure une réelle dynamique pour certains groupes qui avaient, qui étaient déjà dans l'objectif attendu : échanger des informations à collecter sur le binôme pour l'identifier. C'était vraiment très intéressant ».

Sans nier les difficultés techniques, il fait valoir que celles-ci ont obligé les élèves à chercher d'autres solutions et à s'entraider, cela les a donc placés dans une position dynamique et en autonomie. De plus, ils ont pu remplir l'objectif pédagogique sans se décourager.

Cet argument amène un nouveau thème : la motivation des élèves. Cela permet de replacer les élèves et leur réussite au centre des préoccupations. Le thème est repris par plusieurs intervenants, $y$ compris des enseignants, qui se rallient progressivement : "j'étais étonné », "ils ont quand même avancé un peu », «c'est vrai », « on l'a observé ce matin, les élèves sont restés motivés ».

Enfin, l'ingénieur reprend et se rallie à la proposition de la mise en place d'une formation, insistant à plusieurs reprises sur la faisabilité et l'efficacité de cette solution: "ça se débloque très facilement avec une formation », « très volontiers », « très facilement $»$.

\section{Objet frontière et brokering}

Dans cette séquence, la traduction initiée se poursuit en intégrant dans l'objet la composante pédagogique et une nouvelle composante, plus didactique concernant les apprentissages des élèves. Celle-ci est apportée par la remarque du référent du comité de pilotage des LéA citée dans le paragraphe précédent, qui constitue pour la suite de la réunion un acte de brokering décisif en élargissant la frontière de l'objet frontière collège virtuel.

Les discussions sur les possibilités pédagogiques liées aux potentialités techniques entament ainsi une action de traduction en reliant les propriétés des composantes pédagogique et techniques de l'objet frontière et leurs interprétations dans les différentes 
institutions présentes. L'action de transfert engagée uniquement sur la dimension technique est ainsi dépassée pour atteindre la composante pédagogique de l'objet. Les éléments de dialogue qui suivent énoncés par le chercheur montrent cette modification de l'action sur l'objet frontière :

« En même temps, la scénarisation de ce qui s'est passé, comment les élèves pouvaient se retrouver enfin parce que moi ce que, ce que j'ai observé, en regardant, oui j’ai regardé un petit peu, dans la classe, c'est que les élèves ne savaient pas exactement à qui ils parlaient. Et donc, donc, ils parlaient un peu dans le vide et donc c'était une conversation globale, et donc en fait... ben oui... on ne s'entendait pas bien... y avait beaucoup de... oui vas-y ».

Il y a là un retour vers la composante pédagogique dans une action de traduction : quel sens peut-on donner à la scénarisation, qui est une souscomposante pédagogique? Comment cette souscomposante peut-elle inférer des propriétés de la composante pédagogique du collège virtuel?

Enseignant 1: «oui c'est parce qu'il fallait passer d'un groupe à l'autre et expliquer par exemple pour le [groupe de l'école] $n^{\circ} 2$, discuter avec le groupe $\mathrm{n}^{0} 2$ et expliquer en particulier ».

Enseignant 2: « voilà on tchate, on tchate ».

La proposition de discussion sur la scénarisation dans la composante pédagogique est acceptée et mise en relation avec ce qui s'est passé dans la matinée.

Enseignant 1: «Et du coup, nous, on se retrouvait avec une limite de contraintes, écrire en anglais, donc là déjà c'est pas facile mais ensuite c'est un départ pour les CM1-CM2. Donc...»

Ingénieur : «Par contre, ça, ça peut se faire à l'oral aussi ».

La composante pédagogique est remise en relation avec la composante technique. La zone frontière du collège virtuel s'élargit suite à cette action de traduction. Dans cette séquence l'action de brokering a une conséquence immédiate sur l'évolution de la discussion; on peut penser que c'est parce que le transfert précédent a été suffisamment abouti que la possibilité d'agir différemment sur l'objet frontière est acceptée par les acteurs, ce qui permet cet élargissement.

\section{Séquence de clôture}

\section{Analyse de l'interaction}

Au cours de la suite de la discussion, un accord tacite est alors trouvé : avec un objectif pédagogique clair, une formation des enseignants et en entretenant la motivation des élèves, des activités peuvent être déployées au sein du collège virtuel. Le chercheur résume les propositions en précisant : « une fois passé les problèmes techniques », anticipant sur l'argument qui risque de revenir.

\section{Objet frontière et brokering}

C'est l'ensemble des discussions qui trouvent ici un épilogue avec la mise à plat d'un consensus trouvé dans la discussion. Les actions sur l'objet frontière sont à ce moment clairement dirigées vers une utilisation pragmatique du collège virtuel dans les pratiques d'enseignement. C'est la suite des observations dans les classes qui permettent ici de confirmer cette dimension pragmatique ébauchée dans cette phase finale; ces éléments sont abordés dans la discussion.

\section{Schéma général}

Alors que l'intervention initiale du chercheur suggérait un format d'interaction présenté sur le schéma 1 ( $\mathrm{A}, \mathrm{B}, \mathrm{C}$ concernant les thèmes des interventions respectivement sur les usages passés, du matin et futurs), le format effectivement observé fait une place importante aux difficultés techniques (Schéma 2, D pour les interventions concernant ces difficultés et ${ }^{*} \mathrm{C}$ pour l'absence du thème $\mathrm{C}$ ).

Schéma 1 : Format d'interaction initié par le chercheur

$$
\begin{aligned}
& \text { I - Intervention initiative : «Expliquez-nous quels } \\
& \text { sont vos usages passés (A), vécus ce matin (B) et } \\
& \text { envisagés pour le futur (C) ?». } \\
& \text { II - Interventions réactives: } \\
& \text { - A, B, C } \\
& \text { - A, B, C « usages» } \\
& \text { - A, B, C }
\end{aligned}
$$

En effet, au cours de l'interaction, on constate que si le déroulé proposé est accepté dans un premier temps, la discussion reste longuement 
focalisée ensuite sur les difficultés techniques des enseignants, ces derniers ayant fait l'expérience des difficultés matérielles et techniques liées à l'utilisation du collège virtuel en classe. La négociation se joue autour du thème «technique » et empêche la complétude du déroulé proposé puisque, comme le disent les enseignants, « les ambitions pédagogiques sont contraintes par la réalité technique ». C'est grâce à l'écoute et à la prise en compte des arguments des uns et des autres que la discussion avance et aboutit au thème initialement proposé : les usages pédagogiques possibles pour un projet en construction (schéma 2).

Schéma 2 : Format d'interaction effectivement observé

$$
\begin{aligned}
& \text { I - Intervention initiative : "Expliquez-nous quels } \\
& \text { sont vos usages passés (A), vécus ce matin (B) et } \\
& \text { envisagés pour le futur (C)? ». } \\
& \text { II - Interventions réactives : } \\
& \text { - A, B, C + D usages et difficultés techniques } \\
& \text { - A, B + D }\left({ }^{*} \mathrm{C}\right) \\
& \text { - A, B + D }\left({ }^{*} \mathrm{C}\right) \text { usages passés et vécus et }
\end{aligned}
$$

difficultés techniques

- A, B + D ( $\left.{ }^{*} \mathrm{C}\right) \quad\left({ }^{*}\right.$ usages futurs $)$

III. Suite à la négociation et à l'argumentation

- A, B, C D

- A, B, C D

- A, B, C D

L'enjeu était ici important puisqu'il s'agissait de partenaires potentiels qui n'étaient pas encore formellement engagés dans le projet. La prise en compte des arguments de tous parait de toute façon nécessaire à l'avancée productive du travail de conception collaborative, passant d'activités de transfert sur l'objet frontière, à des activités de traductions, qui pourront amener des transformations dans le domaine pragmatiques des pratiques des différents acteurs.

L'analyse des verbatim de la réunion fait apparaitre les actions réalisées sur l'objet frontière collège virtuel qui est au cœur des préoccupations des acteurs de cette réunion. Ces actions conduisent à un élargissement de la zone frontière de l'objet frontière pour tous les acteurs. Elles sont rendus possibles par l'acceptation du contrat proposé initialement mais aussi, par les perspectives ouvertes pour résoudre les problèmes considérés comme suffisamment importants pour être maintenus comme sujet légitime de l'action. Le brokering apparaît alors de deux façons, d'une part pour faciliter une action de transfert sur l'objet frontière en permettant aux acteurs de mieux comprendre le fonctionnement même de l'objet et de percevoir les pistes de résolution des problèmes évoqués, et d'autre part pour engager une action de traduction dont l'effet est un élargissement de la zone frontière visible, acceptable par tous les acteurs.

\section{Réunion du projet École Numérique et Industrie}

Cette réunion se situe trois mois après le début du projet et réunit quatre enseignants, un enseignantchercheur spécialiste des STI (Sciences et Techniques industrielles), un ingénieur pédagogique et une chercheuse. L'objectif est de concevoir la scénarisation et la métaphore du jeu, c'est-à-dire quels défis seront à réaliser par le joueur et dans quel objectif. Le jeu en cours de conception est l'objet sur lequel l'ensemble $\mathrm{du}$ groupe travaille. Nous le considérons comme l'objet frontière entre les différents participants. Lors d'une précédente réunion, il avait été évoqué la possibilité que le jeu consiste à mener une enquête suite à une explosion qui avait eu lieu dans une usine de fabrication de robots.

L'extrait étudié s'est déroulé lors de la troisième rencontre entre ces participants, ils ont donc déjà eu l'occasion d'échanger ensemble auparavant. Lors de cette rencontre, les participants ont travaillé ensemble toute la journée sous la forme de deux sessions de deux heures (le matin et l'après-midi). L'extrait se situe une demi-heure après le commencement de la session de l'après-midi. La consigne qui a été donnée en ouverture par l'ingénieur pédagogique est la suivante: " en utilisant les tableaux, le but c'est de faire des petites cases et de raconter l'histoire du jeu et de voir comment l'enquête peut s'inscrire dedans ». Cette interaction se découpe en deux séquences principales.

\section{La proposition initiale et le désaccord explicite}

\section{Analyse de l'interaction}

Au début de l'extrait étudié, l'enseignant-chercheur spécialiste des STI (EC1) fait une proposition : pourquoi ne pas centrer l'enquête autour du cycle de 


\section{OBJETS FRONTIÈRES ET BROKERING DANS LES NÉGOCIATIONS EN RECHERCHE ORIENTÉE PAR LA CONCEPTION}

Réjane Monod-Ansaldi, Caroline Vincent E Gilles Aldon

vie d'un produit de l'usine? Il fait valoir différents arguments et précise son idée.

Immédiatement, El, enseignant d'histoire-géographie claque la langue, penche sa tête sur le côté puis exprime son désaccord par une suite d'arguments : « par contre le vocabulaire que tu as utilisé c'est de l'enseignement supérieur [...] je suis pas sûr qu'au collège les gamins les connaissent », " une explosion c'est compréhensible ». ECl argumente à son tour, expliquant que le cycle de vie d'un produit peut paraître complexe mais qu'il pourrait être amené de façon plus simple auprès des collégiens, sous la forme d'une métaphore : «que le scénario soit basé là-dessus mais sans forcément au début leur dire euh on va : étudier le cycle de vie produit c'est en fait le fait qu'ils parcourent le plateau, qu'ils découvrent ah oui bah tiens quand je fais euh sur cette partie-là du plateau, on est en train de me parler de comment le produit euh :: -il a été conçu etc., sans leur dire au début $»(E C 1)$.

Il fait ainsi valoir que le cycle de vie d'un produit serait métaphorisé dans le jeu et non pas explicite. On retrouve de nouveau cet argument dans cette intervention métadiscursive : « c'était ma question de début ça veut dire est ce qu'on définit ce que va avoir l'utilisateur final là ou est-ce qu'on définit c'qu'est c'qu'est caché derrière? » (EC1).

Le désaccord est verbalisé de façon explicite : « je pense qu'il faut rester sur l'explosion », « pour moi on est en train de trop intellectualiser le truc» (E1).

Durant cette première séquence, seuls ces deux participants échangent leurs arguments durant une vingtaine de tours de parole, les autres écoutent.

Composantes de l'objet frontière discutées dans le désaccord explicite

La proposition de scénarisation de ECl autour du cycle de vie d'un produit peut être regardée comme une tentative de faire entrer dans le jeu un nouveau savoir de STI. D'un point de vue didactique, il s'agit d'une proposition de transposition d'un savoir de référence de STI vers un savoir à enseigner ou à travailler par le jeu. Du point de vue de la conception de jeu, cette proposition est bien en lien avec l'objectif de la réunion, qui concerne la métaphore du jeu et le scénario, dont le choix amène à relier les aspects ludiques et didactiques : comment choisir une situation de jeu qui permette effectivement aux élèves de travailler certains savoirs? Comment faire pour que cette situation soit effectivement ludique? Ainsi, le travail proposé sur les composantes didactique et ludique (scénario du jeu) amène ECl à prendre en compte une composante scientifique, en lien avec les savoirs de référence, qu'il représente en tant qu'expert des STI, appartenant à l'institution universitaire.

L'expression du désaccord d'El concerne ce savoir de référence, « c'est de l'enseignement supérieur », qu'il identifie mais rejette comme trop difficile pour les élèves de collège. Pour lui, la transposition didactique proposée, n'est pas légitime ou pertinente, « c'est trop intellectualisé », ce savoir de référence n'est pas un objectif d'apprentissage recevable. Il introduit ainsi dans l'interaction une autre composante didactique de l'objet frontière, correspondant au savoir à apprendre par les élèves, en lien avec leurs connaissances propres ( «je suis pas sûr qu'au collège les gamins les connaissent ») et s'intéresse au registre de vocabulaire compréhensible par les élèves. En tant qu'enseignant au sein de l'institution « collège », il défend une composante de l'objet dont il est un spécialiste ici : l'adaptation des objectifs d'apprentissage aux capacités des élèves. C'est comme s'il refusait de faire entrer cette composante de l'objet dans la zone frontière.

On peut également faire l'hypothèse ce savoir « trop intellectualisé » lui parait difficile à faire entrer dans le jeu en gardant un aspect ludique, et que c'est pour cela qu'il préfère l'explosion, car « c'est simple et clair ». Il mobilise alors également la composante ludique, c'est à dire la métaphore du jeu en construction.

EC1, qui paraît conscient que le concept de transposition de savoirs dans le jeu n'est pas pris en compte par E1, tente de relier les composantes ludique et scientifique, en reformulant de plus en plus précisément le processus de transposition, jusqu'à dire « est ce qu'on définit ce que va avoir l'utilisateur final là [la métaphore], ou est-ce qu'on définit c'qu'est caché derrière [les savoirs en jeu] ? ». Ces éléments sont saisis transitoirement par El qui acquiesce quand ECl indique « du moins ça peut pas être présenté comme ça aux collégiens au début », avant de revenir à une posture d'opposition.

Ainsi, le désaccord explicite observé peut être analysé comme la coexistence simultanée dans le débat de plusieurs composantes de l'objet frontière portées ou convoquées par des acteurs d'institutions différentes, se sentant chacun responsable de les faire exister dans l'objet. El regarde la compo- 
sante ludique, ECl la composante des savoirs scientifique à placer dans le jeu, et même si ECl propose des pistes pour articuler les deux composantes, E1 ne prend pas réellement en compte la composante des savoirs. Les deux protagonistes ne regardent finalement pas la même composante de l'objet, la zone frontière d'intersection entre les composantes identifiées n'est pas suffisamment large pour qu'ils puissent parler ensemble du même objet. À ce moment, leurs regards ne se rencontrent pas, le transfert ne fonctionne pas, l'objet jeu n'est pas véritablement pour eux un objet frontière.

\section{Intervention d'un médiateur et ralliement des autres participants}

\section{Analyse de l'interaction}

Au début de cette séquence, l’ingénieur pédagogique intervient en reformulant le point de désaccord : « ce qui te gêne c'est rentrer dans un processus de cycle de vie, on est dans un processus projet ou entreprise », ratifié par l'enseignant « oui c'est trop ancré dans la réalité ».

Les deux parties échangent alors de nouveaux arguments sans trouver d'accord. L'ingénieur pédagogique reformule le point de vue de ECl puis affiche explicitement sa neutralité : " par contre je ne défends le cycle de vie ou hein » en secouant les mains devant lui. Il est ratifié par les deux parties « on est bien d'accord ». Il prend ainsi le rôle dans l'interaction de médiateur neutre.

Le désaccord s'est cristallisé, chacun des autres enseignants participe à son tour au débat, cherchant un compromis. Ils reprennent les arguments de El pour lui proposer des solutions. Prenons l'exemple de cet argument : « je ne vois pas comment faire rentrer ça dans les programmes ». Auquel sont proposées les solutions suivantes : «ben tu feras la logistique en géo » (E3), « ça peut rentrer dans la durabilité » (E2), « si tu prends l'aspect besoin définition des besoins » (EC1).

Durant ces échanges, le médiateur propose des reformulations et demande aux uns et aux autres des précisions, prenant en notes leurs réponses au tableau.

Malgré ces propositions, l'enseignant reste en désaccord : «ça me parle moins », «moi j'ai un programme à faire ».
L'extrait étudié s'achève ici, cette discussion en suspens restera un fil rouge des réunions suivantes, en attendant un accord.

Objet frontière et brokering dans la discussion des deux points de vue opposés à l'échelle du groupe

En reformulant le désaccord « ce qui te gêne c'est rentrer dans un processus de cycle de vie, on est dans un processus projet ou entreprise », l'ingénieur pédagogique fait apparaître une nouvelle composante, celle des pratiques de référence dans l'entreprise. Il amène ainsi l'idée que le « cycle de vie d'un produit » n'est pas seulement un savoir universitaire, mais qu'il correspond à une façon de faire et de penser dans l'entreprise, qui représente encore une autre institution. Cette dimension est reconnue, mais non acceptée par E1, qui reproche au cycle de vie d'être «pas assez terre à terre » (c'est à dire trop intellectualisé ou universitaire), puis « trop ancré dans la réalité » (c'est à dire de correspondre à une composante de la STI en lien avec les pratiques de références) quand l'ingénieur pédagogique lui pointe ses contradictions « ou trop terre à terre plutôt, non?». Ainsi, soit El ne peut pas prendre en compte la transposition didactique de savoirs et de pratiques sociales de référence dans les savoirs à enseigner, soit il ne lui parait pas pertinent de le faire, par rapport au cadre institutionnel des savoirs scolaires à enseigner, ou par rapport au potentiel ludique de ce savoir pour le jeu. La zone frontière de la composante des savoirs à intégrer dans le jeu n'est pas partagée par tous : EC place le jeu dans une culture STI alors que El considère le jeu avec des intentions de transmission de savoirs liés à sa discipline en dehors du contexte STI.

Par sa neutralité affichée, ses reformulations, sa façon de pointer certaines contradictions, ses questions qui favorisent l'expression de différents point de vue, et par l'identification explicite de certaines composantes de l'objet, l'ingénieur pédagogique qui intervient en médiation déclenche un épisode de brokering permettant à l'ensemble du groupe de se positionner et de travailler les différentes composantes de l'objet frontière mobilisées par les deux premiers protagonistes. En effet, suite à ses interventions, six personnes produisent des interventions concernant les savoirs à intégrer dans le jeu, trois interviennent au sujet des pratiques de références, trois font référence à la métaphore du jeu, et cinq explorent la question de la relation de ce savoir universitaire, « le cycle de vie d'un produit», 
Figure 1 : Schéma construit au tableau par l'IP durant la séquence reliant les éléments ludiques et scientifiques dans le terme théorique de métaphore

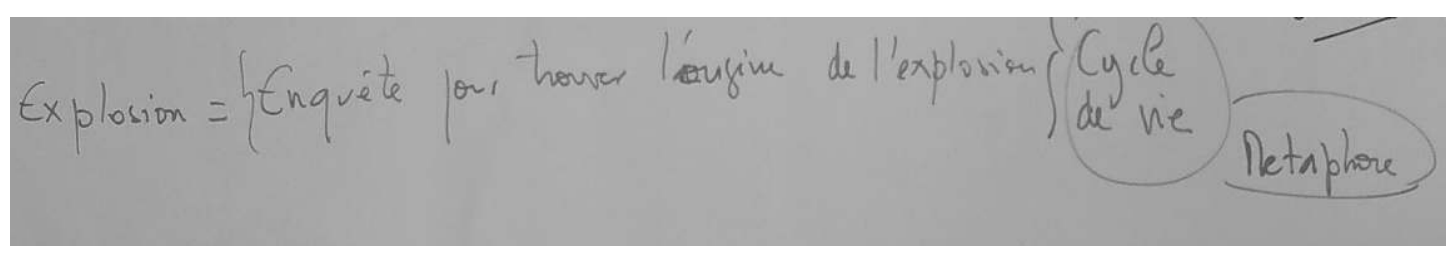

avec les programmes d'enseignement. Le fait que le broker écrive au fur et à mesure sur le mur les éléments discutés au cours de la séquence, permet à tous d'identifier les différentes composantes de l'objet frontière jeu présentes dans les échanges, et favorise le regard de chacun. Sur le schéma qu'il construit de gauche à droite durant cette séquence (figure 1) on retrouve les éléments des deux composantes qui coexistaient difficilement dans la première séquence : explosion et enquête [éléments métaphoriques retenus par El dans la composante ludique], puis cycle de vie [savoir à intégrer dans le jeu proposé par ECl dans la composante scientifique] puis métaphore [référent théorique de la composante ludique]. On peut ainsi penser que le brokering permet au groupe de mener une activité de transfert autour de l'objet jeu, sans pouvoir obtenir un plein transfert concernant la composante savoirs à intégrer dans le jeu. Au terme de cette réunion, les composantes savoirs à intégrer dans le jeu et métaphore $d u$ jeu sont mises en relation par presque tous les participants. On peut penser qu'une discussion plus sémantique sur leur articulation pourra s'ébaucher par la suite, dans une activité de traduction.

\section{Schéma général de l'interaction}

Le schéma ci-dessous propose une décomposition synthétique des différents mouvements observés au cours de cette négociation.

Dans cet extrait, le désaccord est explicite et n'aboutit pas immédiatement à un compromis, puisque malgré les efforts collectifs, chacun reste sur ses positions. Dans la version finale du jeu, aucune de ces deux solutions n'a finalement été retenue, une troisième voie a été trouvée $e^{5}$. Ici, le désaccord permet aux participants de prendre des positions et permet de mettre en exergue les éléments qui sont essentiels aux uns et aux autres. ECl veut représenter dans le jeu des façons de faire des STI ancrées dans la réalité et ne souhaite pas un scénario fantaisiste,

Schéma 3 : Schéma général de l'interaction
I. Intervention initiative
ECl - Proposition de scénarisation d'un jeu vidéo : centrer l'histoire sur le cycle de vie d'un produit II. Intervention réactive
E1 - Désaccord explicite argumenté
III. Cristallisation du désaccord
ECl et El échangent leurs arguments sans trouver de compromis.
IV. Intervention du médiateur puis des autres
IP fait reformuler les deux participants, prend des notes au tableau.
Chacun donne son point de vue en cherchant un compromis.

alors que El souhaite embarquer ses élèves avec une histoire plus ludique et qui lui permettra d'aborder le programme scolaire, notamment en histoire-géographie pour ce qui le concerne.

\section{DISCUSSION}

\section{Importance des négociations dans les travaux collaboratifs entre acteurs d'institutions différentes}

L'étude des deux corpus en termes de négociation nous a permis de mettre en exergue les préoccupations de chacun des participants, de rendre compte 
de leurs rôles interlocutifs (la position de médiateur par exemple) et de l'évolution des objets négociés au cours du temps.

Dans les deux cas étudiés, les négociations sont différentes puisque l'une reste implicite alors que l'autre est explicite (le désaccord est verbalisé clairement) et que la première abouti à un compromis alors que la deuxième reste en suspens jusqu'à une prochaine réunion pendant laquelle la discussion reprendra. Ainsi, ce n'est pas l'aboutissement de la négociation qui est la plus importante pour le fonctionnement du groupe et l'engagement des acteurs, mais bien le processus de négociation. En effet, nous avons démontré que ce processus permet aux participants d'échanger des arguments et ce faisant de discuter certaines composantes des objets frontière. Ainsi, ils construisent une culture commune et une compréhension mutuelle nécessaire au travail de conception collaborative.

Dans le cas du premier corpus par exemple, on peut faire l'hypothèse que si les arguments avancés par les enseignants n'avaient pas été entendus et n'avaient donc pas donné lieu à une négociation, ces derniers se seraient peut être désengagés du projet en devenir. Cette interaction leur a permis de faire entendre leurs contraintes contextuelles et professionnelles, indissociables du projet pédagogique futur et dont les autres participants n'avaient pas forcément conscience. La négociation a ainsi fait évoluer la proposition initiale en y incluant la prise en compte des contraintes techniques et matérielles. Dans le cadre du projet ENI, des entretiens individuels menés avec les participants en aval des réunions de conception nous ont appris que deux des enseignants avaient senti une démotivation temporaire à l'égard du projet. Pour eux, ce manque d'intérêt est venu d'une décision collective avec laquelle ils n'étaient pas d'accord : l'élaboration d'un plateau de jeu tangible en plus du plateau de jeu virtuel.

Pour l'enseignante E2, ce choix ne paraissait pas compatible avec ses propres contraintes :

« Je pense que c'est sans doute pratique pour certains profs ou certains élèves, peut-être qu'il y a ce besoin physique. Moi sur des collégiens, je vois pas comment je l'utiliserais parce que si on est sur une salle info, je vois pas comment le déplacer, remettre tout, c'est plutôt quelque chose qui est voué à être perdue $[. .$.$] . Donc en fait j'arrivais pas moi avec mes$ contraintes professionnelles de voir l'intérêt d'avoir ce plateau » (E2).

L'usage de ce plateau tangible ne paraissait pas cohérent avec le projet global pour l'enseignant E3 :

«À un moment donné je pense que je me suis un peu perdu dans le projet. [...] Autant au début j'arrivais bien à cerner et à voir qu'on avait cette idée qu'on avait cette enquête qui allait permettre de faire rentrer l'élève dans la culture scientifique. Après, quand on a été sur le jeu de plateau qu'on allait devoir mener en même temps alors là je reconnais que je... Là j'ai un peu perdu le fil quoi » (E3).

En visualisant les enregistrements de l'ensemble des réunions, on s'aperçoit que leur désaccord n'a jamais été exprimé avec le groupe. Au contraire, ils ont pris une part active dans la conception du plateau de jeu, favorisant l'intérêt commun plutôt que le leur, ce que confirme l'enseignante E2 : «j'ai essayé de faire, je voyais pas toujours les tenants et les aboutissants parce que j'ai pas toujours les mêmes types d'élèves. Mais j'essayais de le faire » (E2).

Il est intéressant de constater qu'un point de désaccord qui n'est pas exprimé et donc qui n'a pas l'opportunité de donner lieu à une négociation, peut créer une baisse de l'engagement des participants, sans que l'équipe en soit consciente. Cela montre l'importance de la fonction des désaccords et des processus de négociations dans les réunions de conception collaborative. La question qui se pose pour les animateurs de projet est donc de savoir créer les conditions d'expression des désaccords au cours des réunions, à l'aide d'entretiens ou de questionnaires individuels ou lors de moments informels pendant lesquelles la parole souvent se libère.

Les réunions de co-conception du projet ENI ont été menées dans le laboratoire d'innovation pédagogique et numérique (LIPeN), où des méthodes, et du matériel particuliers sont développés par des personnes ressources pour favoriser la prise de parole et la créativité des participants. Il paraîtrait judicieux de prendre en compte également l'importance des moments informels en dehors des réunions collectives pour identifier les désaccords larvés qui ne sont pas toujours intelligibles notamment pour l'animateur pris dans l'action. Dans le prolongement de cette recherche, une analyse complémentaire pourrait être 


\section{OBJETS FRONTIÈRES ET BROKERING DANS LES NÉGOCIATIONS EN RECHERCHE ORIENTÉE PAR LA CONCEPTION}

Réjane Monod-Ansaldi, Caroline Vincent E Gilles Aldon

menée pour identifier les conditions qui favorisent l'émergence des négociations dans les réunions de conception collaborative.

\section{Objets frontière et brokering dans les négociations}

Nos analyses ont permis de repérer différentes composantes de l'objet frontière au sein des séquences de négociation, et de caractériser des activités de transfert, traduction ou transformation sur certaines de ces composantes durant la collaboration. Les regards portés par chacun des acteurs sur un ensemble commun de composantes de l'objet (transfert) apparaissent déterminants pour la mise en place d'échanges effectifs concernant le sens et l'articulation de ces composantes (traduction), qui pourra permettre par la suite le développement de travaux pragmatiques de conception collaborative autour de l'objet et l'évolution des pratiques reliées (transformation).

Les obstacles à ces activités apparaissent quand des composantes portées par une communauté ne sont pas vues par une autre. Le broker joue alors un rôle crucial pour la poursuite des travaux, en identifiant ces composantes « aveugles », et en attirant l'attention du collectif sur elles, par des questions, qui permettent aux différents points de vue, issus d'institutions différentes, de s'exprimer de façon légitime. Il intervient par des reformulations qui proposent de nouvelles pistes pour entendre ces points de vue et regarder les différentes composantes, et par des représentations qui permettent de visualiser ces composantes au sein du même espace, et d'ébaucher des liens entre elles.

Outre son potentiel heuristique de compréhension des travaux collaboratifs, ce modèle reliant objet frontière et brokering nous parait avoir une portée pragmatique pour les animateurs de projets collaboratifs. En effet, repérer les composantes mentionnées par les différents locuteurs d'une interaction collaborative, et en particulier celles qui ne sont pas considérées par tous, peut permettre d'identifier les obstacles potentiels ou à l'œuvre, et de proposer des pistes pour favoriser les activités de transfert, traduction ou transformation en cours, par des actions de brokering. Nous explorons actuellement ces potentialités en mobilisant ces concepts pour l'analyse de pratiques dans des formations destinées à des personnes animant ou pilotant des travaux de recherche collaborative, tels que les correspondants des Lieux d'éducation associés à l'IFE (LéA), les chercheurs, les cadres de l'Éducation Nationale ou les formateurs d'enseignants.

\section{Collaboration, négociation, conception de dispositifs numériques et construction de savoirs}

Dans la réunion étudiée du projet Pédagogies Immersives, la négociation a permis la prise en compte des contraintes techniques et matérielles par les chercheurs, mais aussi la prise en compte des propositions didactiques par les enseignants. Les questions discutées ont constitué le point de départ du travail mis en œuvre dans le collège virtuel. Ainsi, les enseignants se sont saisis des propositions de scénarisation et ont construit avec leurs élèves un jeu de découverte des 7 merveilles du monde. Les actions sur l'objet frontière ont ainsi élargi sa compréhension dans une chaîne d'actions de transfert, débouchant sur une prise en compte des difficultés à surmonter, de traduction, débouchant sur des propositions d'utilisation du collège virtuel à des fins didactiques, et de transformation qui ont conduit à la mise en œuvre effective de projets didactiques scénarisés mobilisant le contexte particulier du collège virtuel et ses propriétés. L'analyse des travaux menés dans la suite du projet confirme ainsi que les actions sur l'objet frontière acceptées à travers la négociation permettent l'internalisation de praxéologies, et aboutissent à la production de séquences pédagogiques satisfaisant à la fois des exigences didactiques et la réalité des classes et de la plateforme. De plus, les analyses didactiques des apprentissages mathématiques des élèves dans ces situations, montrent que la réalité augmentée présente dans le collège virtuel permet de construire un espace intermédiaire entre le monde réel et l'abstraction mathématique qui favorise l'appropriation des concepts en jeu (Aldon $\&$ Raffin, 2019).

Le jeu obtenu en fin de projet ENI est décrit dans le bilan (Benech \& Vincent, 2017, p. 49-66). La métaphore choisie repose sur un incident (non pas une explosion, mais un piratage) et certains éléments du cycle de vie des produits sont représentés par la figuration dans le scénario de différents métiers liés au produit et dans les quatre zones du plateau de jeu 
représentant la sphère administrative, la production, la recherche-développement et l'activité de commercialisation et de service après-vente (figure 2). Ainsi, les composantes identifiées durant la négociation étudiée ont été retravaillées au sein du groupe et sont intégrées dans le jeu. La production finale du projet tient donc compte à la fois des points de vue du chercheur et des enseignants, dans une perspective de praxéologie partagée aboutissant à un prototype de jeu à la fois riche du point de vue des savoirs visés et effectivement utilisable dans les classes.

Lors de la première année de fonctionnement du projet, l'effort a été concentré sur l'élaboration des fondements de la ressource, la conception d'un prototype de jeu et sur le processus de conception collaborative. À plus long terme, les questions scientifiques concernent l'appropriation du jeu par les enseignants et les élèves, sa jouabilité et son potentiel didactique.

Dans les deux cas étudiés, les situations de classe ou le jeu numérique conçus tiennent donc compte des contraintes et des visées des différents acteurs impli- qués dans la conception, ce qui leur permet à la fois d'être utilisable en classe, d'embrasser des visées didactiques ambitieuses et de tenir compte des cultures de références dans la sphère sociale. Cette co-conception menant à un partage de praxéologie par les choix et les actions négociés au sein du groupe à chaque étape, permet de produire et d'étudier des dispositifs d'apprentissage s'insérant dans le système complexe de la classe. (Sanchez \& Monod-Ansaldi, 2015.) En effet, pour Morin (1990) l'action, en tant que stratégie, « suppose la complexité, c'est à dire aléa, hasard, initiative, décision, conscience des dérives et des transformations » (p. 107). Elle oblige à « faire avec» les différents paramètres du système dans lequel elle se déploie, sans les isoler. La recherche orientée par la conception allie à sa flexibilité d'adaptation de l'action aux variabilités et inattendus du contexte, une analyse fine des adaptations réalisées et de leurs impacts, et une régulation de leur cohérence avec les visées globales de la recherche. Le travail en conditions écologiques, dans des classes avec des élèves et des enseignants, oblige à tenir compte des contraintes

Figure 2 : Plateau du jeu produit en fin de projet

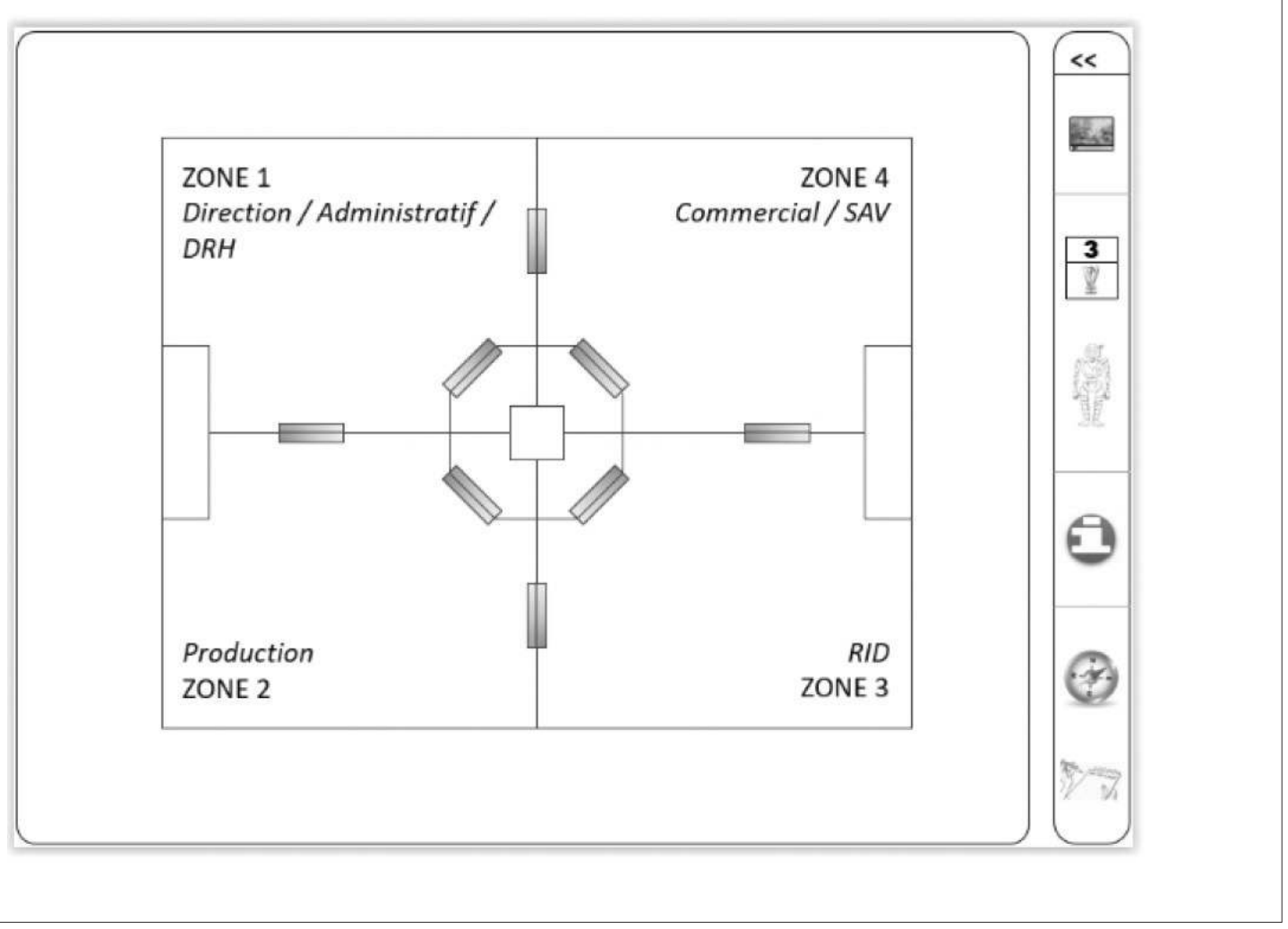




\section{OBJETS FRONTIÈRES ET BROKERING DANS LES NÉGOCIATIONS EN RECHERCHE ORIENTÉE PAR LA CONCEPTION}

Réjane Monod-Ansaldi, Caroline Vincent E Gilles Aldon

portées par chacun des participants, dans les négociations autour des actions à mettre en œuvre. Ainsi, les objectifs de production de dispositifs et de savoirs des recherches orientées par la conception ne pourraient être atteints sans la contribution de chacun des différents acteurs. Les ingénieries coopératives basent également leur fondement théorique sur la nécessité épistémologique de l'action conjointe pour construire un collectif au sein duquel « les chercheurs doivent apprendre des agents de l'action, le jeu qu'ils jouent » (Sensevy, 2016, p. 229). D'ailleurs, l'analyse fine de ce type d'ingénierie coopérative entre enseignants et chercheurs menée par Morellato (2017) en mobilisant le cadre de l'action conjointe (Sensevy, 2011) montre comment après un certain temps, chacun est à même de réorienter l'action didactique collective et de participer à l'élaboration de praxéologies.

\section{CONCLUSION ET PERSPECTIVES}

Le travail présenté ici visait à croiser différents cadres théoriques et différents points de vue pour une meilleure compréhension des processus à l'œuvre dans les travaux collaboratifs de recherche orientée par la conception. Nous avons pu constater l'intérêt de porter sur les corpus des regards croisés depuis l'intérieur et l'extérieur du projet. En effet, la connaissance fine de l'historique du projet, des acteurs impliqués, et de la trajectoire de chacun au sein du projet permet à la personne menant l'analyse interne d'interpréter plus finement certains blocages, certaines avancées, et de replacer les processus en cours dans une évolution sur un plus long terme. Le positionnement extérieur apporte un éclairage complémentaire, faisant ressortir des faits marquants, débusquant de nouveaux questionnements, pointant l'attention sur certains indices parfois ignorés depuis l'intérieur. Ce croisement de regards permets de lever des implicites et de mieux comprendre les collaborations. Les travaux du CrAC montrent d'ailleurs une compréhension meilleure quand les analyses sont réalisées par des personnes de statuts différents (chercheurs, enseignants et formateurs impliqués ou non dans le projet étudiés).

Ces analyses permettent d'éclairer le moteur des transformations des praxéologies des différents acteurs et mettent en évidence la dynamique décrite dans le cadre de la transposition méta-didactique. Ainsi le repérage des composantes de d'objet fron- tière et des actions de brokering au sein des négociations, permet de saisir l'évolution des partages, et de montrer comment les processus d'internalisation et de construction de praxéologies partagées peuvent influer sur la construction collective de savoirs.

Ainsi, dans les réunions de co-conception, les différents points de vue représentés par les acteurs de statuts et fonctions différentes permettent de faire exister dans les travaux de recherche orientée par la conception, différentes composantes de l'objet frontière travaillé. Chaque acteur détient alors la responsabilité de faire vivre dans les interactions, et prendre en compte dans le projet de conception les composantes de l'objet que sa position particulière lui permet de considérer en poursuivant ses propres objectifs professionnels au sein de son institution d'origine. La composition pluri-catégorielle du groupe permet ainsi d'aborder la complexité d'un objet en articulant différents points de vue dans les négociations qui se jouent pour défendre, discuter et relier les différentes composantes de l'objet. Les liens identifiés entre ces composantes deviennent eux-mêmes des composantes de l'objet partagés par le groupe, et peuvent constituer des savoirs nouveaux sur l'objet, qui n'auraient pu être construits hors de la mosaique de regards assurée par l'approche collective. Le fait que les projets de recherche orientée par la conception visent effectivement la production d'un élément pragmatique et son expérimentation dans des contextes authentiques de travail de certains des participants, implique que les processus de transfert et de traduction sur l'objet aboutissent effectivement à des transformations, médiées par des négociations, pour que l'objet co-conçu soit véritablement utilisable dans les pratiques de chacun, qui s'en trouvent transformées.

Des analyses complémentaires doivent cependant encore être menées pour affiner le modèle de description des situations de conception collaborative proposé. De ce point de vue, le cadre de la valuation proposé par Dewey (2011) peut également être mobilisé pour identifier les phénomène à l'œuvre dans le brokering, en reliant les dimensions individuelle et collective des activités de transfert, traduction et transformation sur l'objet frontière travaillés par le groupe (Nizet, Monod-Ansaldi, Aldon, Prieur $\&$ Criquet, 2018).

Les analyses des corpus présentées dans cet article montrent l'importance des négociations dans la mise en place d'un travail collaboratif suffisamment riche pour faire émerger, aussi bien pour les enseignants 
que pour les chercheurs, des savoirs qui n'auraient pas pu être appréhendés sans ces regards croisés sur un objet. Dans le prolongement de cette recherche, une analyse complémentaire pourrait être menée pour mieux identifier les conditions qui favorisent l'émergence des négociations dans les réunions de conception collaborative.

Si l'analyse fine des négociations associée à l'analyse du rôle de broker sur des objets frontières apporte à un niveau local des renseignements importants, la recherche orientée par la conception est un processus long et itératif et qui ne se restreint pas aux interactions au sein de réunions.

Il reste donc nécessaire de mener des analyses longitudinales et de construire ou d'affiner des modèles globaux pour faire le lien entre les événements ponctuels tels qu'analysés dans cet article et l'ensemble des processus à l'œuvre dans le développement de travaux de recherche orientée par la conception.

\section{NOTES}

1. Le contrat de communication peut être définit comme « ce qui fait qu'un acte de communication sera reconnu comme valide du point de vue du sens. C'est la condition pour que les partenaires d'un acte de langage se comprennent un minimum et puissent interagir en co-construisant du sens, ce qui est le but de tout acte de communication » (Charaudeau \& Maingueneau, 2002, p. 138).

2. [https://ife.ens-lyon.fr/fasmed/].

3. [http://ife.ens-lyon.fr/].

4. [http://ife.ens-lyon.fr/lea/outils/outils-administratifs].

5. La métaphore de l'explosion ayant été jugée trop violente par le comité de pilotage du projet, c'est finalement un piratage informatique qui est au cœur de l'enquête. Les différents métiers liés à la fabrication et la commercialisation des robots sont évoqués au fil de l'enquête.

\section{RÉFÉRENCES}

Aldon, G., \& Bécu-Robinault, K. (2013). Modélisation et représentations des états de l'eau par des élèves de SEGPA. Recherches en didactique des sciences et des technologies, 8, 23-46.

Aldon, G., \& Panero, M. (2017). Une classe tablette au collège : Une expérience d'évaluation formative avec la technologie. MathémaTICE, (50) [Repéré à : http:// revue.sesamath.net/spip.php?article857].
Aldon, G., Cusi, A., Morselli, F., Panero, M., \& Sabena, C. (2017). Formative assessment and technology: reflections developed through the collaboration between teachers and researchers. Dans G. Aldon, F. Hitt, L. Bazzini \& U. Gellert (dir.), Mathematics and technology, a CIEAEM sourcebook (p. 551-578). Springer International Publishing [DOI : 10.1007/978-3-319-51380-5].

Aldon, G., \& Raffin, C. (à paraître, 2019). Mathematics learning and augmented reality in a virtual school. Dans T. Prodromou (dir.), Augmented Reality Educational Settings. Melbourne: Sense Publishers.

Artigue, M. (1992). Functions from an algebraic and graphic point of view : cognitive difficulties and teaching practices. Dans E. Dubinsky \& G. Harel, (dir.), The Concept of Function - Aspects of Epistemology and Pedagogy, 25 (p. 109-132). Washington, USA : The Mathematical Association of America.

Astolfi, J.-P. (1993). Trois paradigmes pour les recherches en didactique. Revue française de pédagogie, 103, 5-18.

Arzarello, F., Robutti, O., Sabena, C., Cusi, A., Garuti, R., Malara, N., \& Martignone, F. (2014). Meta-didactical transposition: a theoretical model for teacher education programmes. Dans A., Clark-Wilson, O., Robutti $\&$ N., Sinclair (dir.), The mathematics teacher in the digital era (p. 347-372). Dordrecht, Netherlands : Springer Science -Business Media.

Barab, S., \& Squire, K. (2004). Design-based research: putting a stake in the ground. The Journal of the Learning Sciences, 13(1), 1-14.

Benech, P., \& Vincent, C. (2017). Prototype de resource destiné au développement de la CSTI. Jeu épistémique numérique "Paye ta preuve". Rapport 2017. [Repéré sur le site de l'ENS de Lyon : https://archinfocloud.enslyon.fr/public.php? service=files \& $\mathrm{t}=\mathrm{ea} 7 \mathrm{de} 16 \mathrm{bbc} 75541$ 156722b61c4c98a6f] (PDF).

Brown, A. L. (1992). Design Experiments: Theoretical and Methodological Challenges in Creating Complex Interventions in Classroom Settings. The Journal of the Learning Sciences, 2(2), 141-178.

Bryk, A. S. (2015). Accelerating how we learn to improve. Educational Researcher, 44(9), 467-477. DOI : $10.3102 / 0013189 \times 15621543$

Carlile, P. (2004). Transferring, translating, and transforming: an integrative framework for managing knowledge across boundaries. Organization Science, 15(5), 555-568.

Charaudeau, P., \& Maingueneau, D. (2002). Dictionnaire d'analyse du discours. Paris, France: Editions du Seuil.

Chevallard, Y. (1982). Pourquoi la transposition didactique? Communication présentée au Séminaire de didactique et de pédagogie des mathématiques de l'IMAG, Université scientifique et médicale de Grenoble. Actes de l'année 1981-1982.

Chevallard Y. (1991). La transposition didactique - Du savoir savant au savoir enseigné. Grenoble, France : La Pensée sauvage, deuxième édition augmentée. 


\section{OBJETS FRONTIÈRES ET BROKERING DANS LES NÉGOCIATIONS EN RECHERCHE ORIENTÉE PAR LA CONCEPTION}

Réjane Monod-Ansaldi, Caroline Vincent E Gilles Aldon

Chevallard, Y. (2003). Approche anthropologique du rapport au savoir et didactique des mathématiques. Dans S. Maury \& M. Caillot (dir.), Rapport au savoir et didactiques (p. 81-104). Paris, France : Éditions Fabert.

Collins, A., Joseph, D., \& Bielaczyc, K. (2004). Design research: theoretical and methodological issues. Journal of the Learning Sciences, 13(1), 15-42.

Desgagné, S. (1997). Le concept de recherche collaborative : l'idée d'un rapprochement entre chercheurs universitaires et praticiens enseignants. Revue des sciences de l'éducation, 23(2), 371-393.

Dewey, J. (2011). La formation des valeurs (traduit et présenté par A. Bidet, L. Quéré \& G. Truc). Paris, France : Éditions La Découverte.

Elliott, S. N. (1986). Children's ratings of the acceptability of classroom interventions for misbehavior: findings and methodological considerations. Journal of School Psychology, 24(1), 23-35.

Elliott, S. N., \& Turco, T. L. (1990). Acceptability and effectiveness of group contingencies for improving spelling achievement. Journal of School Psychology, 28(1), 27-37.

Giddens, A. (1987). La constitution de la société. Paris, France : PUF.

Kerbrat-Orecchioni, C. (1990). Les interactions verbales, tome 1. Paris, France : A. Colin.

Kerbrat-Orecchioni, C. (2001). Lanalyse des interactions verbales: la notion de négociation conversationnelle, défense et illustration. Lalies, 20, 63-141.

Kerbrat-Orecchioni, C. (2004). Analyse des conversations et négociations conversationnelles. Dans M. Grosjean \& L. Mondada (dir.), La négociation au travail (p. 17-42). Lyon, France : Presses Universitaires de Lyon.

Lewin, K. (1946). Action Research and Minority Problems. Journal of Social Issues, 2, 34-46.

Maingueneau, D. (1996). Les termes clés de l'analyse du discours. Paris, France : Seuil.

Mallidou, A. A., Atherton, P., Chan, L., Frisch, N., Glegg, S., \& Scarrow, G. (2017). Protocol of a scoping review on knowledge translation competencies. Systematic Reviews, 6, 1-8.

Monod-Ansaldi, R., \& Gruson, B. (2016). Recherches collaboratives dans deux LéA : quels enjeux pour la production de ressources et le développement professionnel des acteurs? Communication présentée au colloque ARCD, Toulouse, France.

Monod-Ansaldi, R., Prieur, M., Arbez, I., \& Golay, A. (2015). Etayer la conception de protocoles expérimentaux par les élèves à l'école primaire. RDST, 12, 113-139.

Morellato, M. (2017). Travail coopératif entre professeurs et chercheurs dans le cadre d'une ingénierie didactique sur la construction des nombres: conditions de la constitution de l'expérience collective (Thèse de doctorat, Université de Rennes, France) [Repéré à : https://tel.archivesouvertes.fr/tel-01591957/document](PDF).
Morin, E. (1990). Introduction à la pensée complexe. Paris, France : Éditions du Seuil.

Munerol, L., Cambon, L., \& Alla, F. (2013). Le courtage en connaissances, définition et mise en œuvre? Une revue de la littérature. Santé Publique, 5(25), 587-597.

Nieveen, N. (2007). Formative evaluation in educational design research. Dans T. Plomp \& N. Nieveen (dir.), An Introduction to Educational Design Research (p. 89-101). Enschede, the Netherlands: SLO Netherlands institute for curriculum development.

Nizet, I., \& Monod-Ansaldi, R. (2017). Construction de bénéfices mutuels en contexte collaboratif : pistes théoriques et méthodologiques. Revue Phronesis, 6(12), 140-152.

Nizet, I., Monod-Ansaldi, R., Aldon, G., Prieur, M., \& Criquet, A (2018). Analyse des processus de valuation dans la production de bénéfices partagés pour les praticiens et les chercheurs partenaires dans des travaux collaboratifs. Communication présentée au symposium 10 ans de recherches menées au sein du réseau RCPE : quels liens entre recherches collaboratives et évaluation? Colloque ADMEE 2018. Luxembourg.

Ridde, V., \& Dagenais, C. (2013). Approches et pratiques en évaluation de programmes. Nouvelle édition revue et augmentée. Montréal, Canada : Presse de l'université de Montréal.

Sacks, H. (1963). On sociological description. Berkeley Journal of Sociology, 8, 1-16.

Sacks H., Schegloff, E. A., \& Jefferson, G. (1974). A simplest systematics for the organization of turn-taking for conversation. Language, 50, 696-735.

Sanchez, E. (2015a). Descriptif du dispositif d'incubateur (LIPn). Livrable du projet ANR JEN Lab.

Sanchez, E. (2015b). Le jeu, espace tridimensionnel. Glossaire de la diversité culturelle à l'ère du numérique. Paris, France : La Documentation française.

Sanchez, E., \& Monod-Ansaldi, R. (2015). Recherche collaborative orientée par la conception. Un paradigme méthodologique pour prendre en compte la complexité des situations d'enseignement-apprentissage. Éducation \& Didactique, 9(2), 73-94.

Sanchez, E., Monod-Ansaldi, R., Vincent, C., \& Safadi, S. (2017). A praxeological perspective for the design and implementation of a digital role-play game. Education and Information Technologies, 22(6), 2805-2824 [DOI : 10.1007/s10639-017-9624-z].

Sensevy, G., Forest, D., Quilio, S., \& Morales, G. (2013). Cooperative engineering as a specific designbased research. ZDM. The International Journal on Mathematics Education, 45(7), 1031-1043.

Sensevy, G., Morales, G., \& Forest, D. (2016). About cooperative engineering: theory and emblematic examples. Educational Action Research, 25(1),128-139.

Sensevy, G. (2011). Le sens du savoir. Éléments pour une théorie de l'action conjointe en didactique. Bruxelles: De Boeck.

Shaffer D. W. (2006). Epistemic frames for epistemic games. Computers and Education, 46(3), 223-234. 
Schön, D. (1993). Le praticien réflexif. À la recherche du savoir caché dans l'agir professionnel. Montréal, Canada : Éditions Logiques.

Star, S. L., \& Grisemer, J. R. (1989). Institutional ecology, 'translations' and boundary objects: amateurs and professionals in Berkeley's Museum of Vertebrate Zoology, 1907-39. Social Studies of Science, 19(3), 387-420.

Star, S. L. (2010). This is not a boundary object: reflections on the origin of a concept. Science, Technology, $\mathcal{E}$ Human Values, 35(5), 601-617.

Tillion, G. (2009). Fragments de vie. Paris, France : Seuil.

Traverso, V. (1999). Lanalyse des conversations. Paris, France : Nathan.

Traverso, V. (2004). Cristallisation des désaccords et mise en place de négociations dans l'interaction : des variations situationnelles. Dans M. Grosjean \& L. Mondada (dir.), La négociation au travail (p. 43-68). Lyon, France : Presses Universitaires de Lyon.

Trompette, P., \& Vinck, D. (2009). Retour sur la notion d'objet frontière. Revue d'anthropologie des connaissances, 3(1), 5-27.

Vinatier, I., \& Morissette, J. (2015). Les recherches collaboratives : enjeux et perspectives. Dans I. Vinatier $\&$ J.-L. Rinatier (dir.), Rencontre entre chercheurs et praticiens : quels enjeux? (p. 137-170). Paris, France : Armand Colin.

Wang, F., \& Hannafin, M. J. (2005). Design-based research and technology-enhanced learning environments. Educational Technology Research and Development, 53(5), 5-23.

Wenger, E. (1998). Communities of practice: learning, meaning, and identity. Cambridge University Press. 